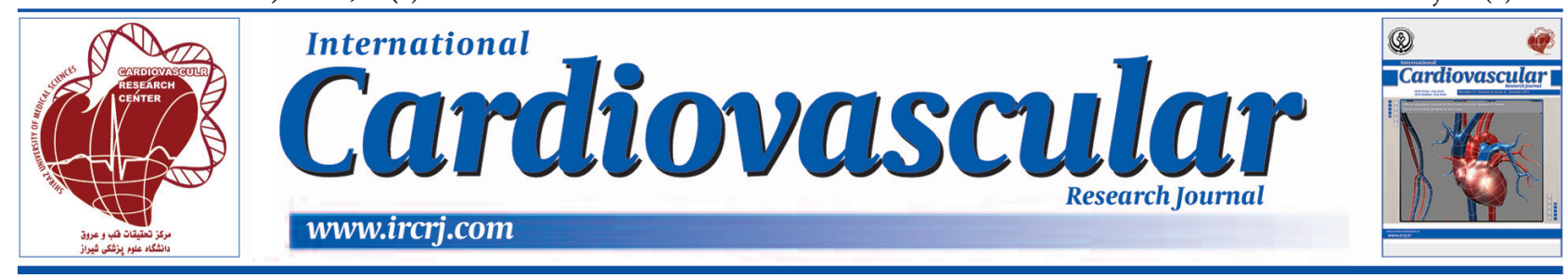

\title{
Coronary Emboli in a Young Patient with Mechanical Aortic Valve: A Rare Cause of Acute Myocardial Infarction
}

\author{
Arash Gholoobi ${ }^{1}$ and Fereshteh Ghaderi ${ }^{1, *}$
}

${ }^{1}$ Atherosclerosis Prevention Research Center, Imam Reza Hospital, Faculty of Medicine, Mashhad University of Medical Sciences, Mashhad, IR Iran

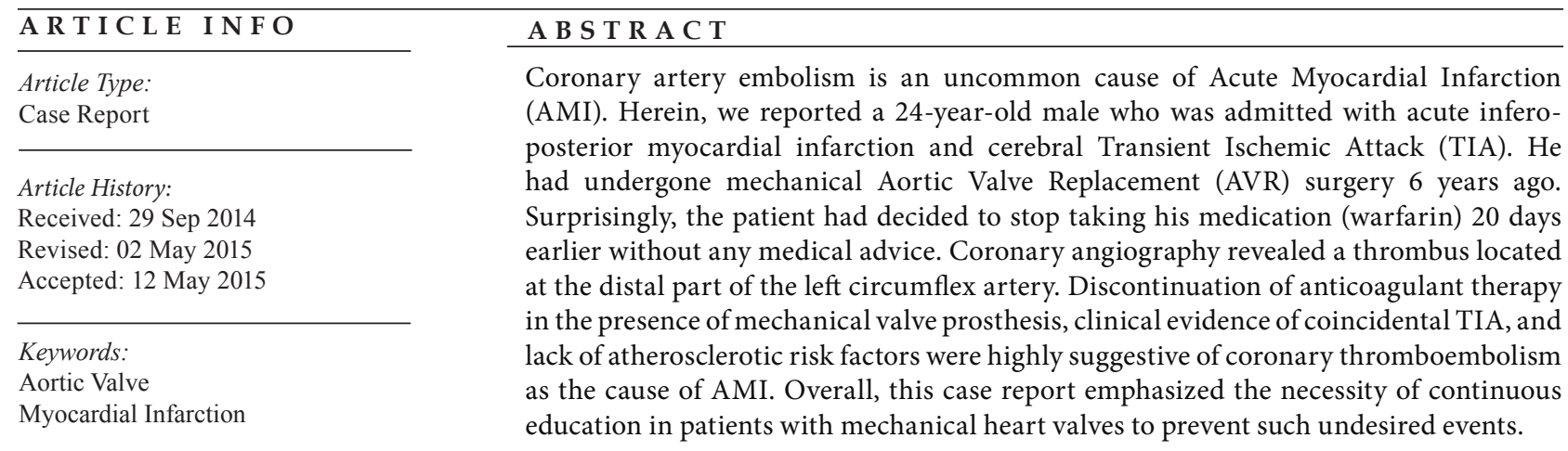

- Implication for health policy/practice/research/medical education:

Information regarding acute coronary embolism in patients with prosthetic heart valves is scarce and based mainly on case reports. Worth noting that our case is unique with respect to the concomitant cerebral transient ischemic attack. In individuals presenting with an acute coronary syndrome, coronary emboli should be kept in mind, especially in the thrombotic state, absence of atherosclerotic risk factors, and coincidence with other embolic events.

\section{Introduction}

Coronary artery embolism is an uncommon cause of Acute Myocardial Infarction (AMI), but it is important to be aware of this entity and its predisposing factors. One of the most important factors is thromboembolism due to mechanical prosthetic heart valves.

Here, we describe a case of thromboembolic Myocardial Infarction (MI) in a young man with mechanical aortic valve prosthesis who decided to discontinue his medications, including aspirin and warfarin, without medical advice.

\section{Case Presentation}

A 24-year-old man was admitted to the emergency department with a chief complaint of typical resting chest pain from a few hours ago. He also had experienced left arm

*Corresponding author: Fereshteh Ghaderi, Atherosclerosis Prevention Research Center, Imam Reza Hospital, Faculty of Medicine, Mashhad University of Medical Sciences, Mashhad, Iran, Tel: +98-9151615412, Fax: +98-5138544504, E-mail:drghaderif@yahoo.com numbness and blurred vision transiently. His past medical history included aortic valve replacement with a bileaflet mechanical prosthesis (St. Jude Medical, Inc., USA) for infectious endocarditis of a bicuspid aortic valve 6 years ago. He had no known atherosclerotic risk factors. However, he had discontinued all his medications, including aspirin and warfarin, upon his own decision since 20 days ago.

On admission, his blood pressure was $130 / 70 \mathrm{mmHg}$ and chest pain relieved. Physical examination was unremarkable with metallic aortic valve sounds clearly heard. Electrocardiography (ECG) showed normal sinus rhythm and new pathologic Q waves in the inferior leads with mild ST segment elevation and $\mathrm{T}$ wave inversion. In addition, the $\mathrm{R} / \mathrm{S}$ ratio in lead $\mathrm{V} 2$ was more than 1 (Figure 1-A) consistent with an acute infero-posterior ST-segment Elevation MI (STEMI) that was reperfused spontaneously. He was.transferred to the coronary care unit and Transthoracic Echocardiography (TTE) was performed, revealing mild hypokinesia of inferior and 

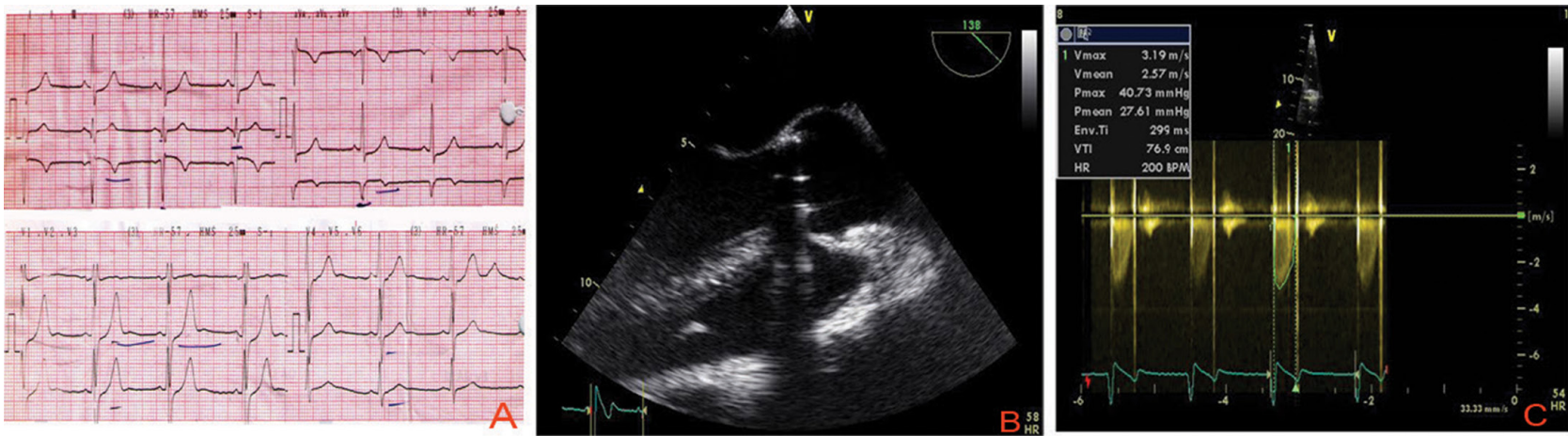

Figure 1. ECG on Admission, Which Shows Recent Infero-Posterior Myocardial Infarction (A), Transesophageal Echocardiography (B, Long Axis Parasternal View) Showing No Apparent Thrombus on the Metallic Aortic Valve, and Doppler Study (C) Indicating an Acceptable Transoartic Gradient for This Specific Type of Prosthesis

posterior walls of the left ventricle, ejection fraction of $50 \%$, and a normally functioning aortic valve with an acceptable trans-valvular peak and mean gradient for the specific type of metallic aortic valve (Figure 1-B, C). Trans-Esophageal Echocardiography (TEE) was also done and neither a dissection flap in the aorta nor a mechanical valve thrombosis was notified (video 1 [to watch the videos, please go to the html format]). There was no other echocardiographic evidence as the source of emboli. Laboratory tests revealed positive cardiac troponin I, elevated creatinine kinase-MB level of 75 $\mathrm{U} / \mathrm{L}$ according to the reference laboratory standards, and International Normalized Ratio (INR) of 1. Other laboratory investigations were unremarkable, including normal fasting plasma glucose and lipid profile. The patient was immediately placed on loading doses of aspirin and clopidogrel in addition to bolus intravenous injection and continuous infusion of weight-adjusted unfractionated heparin together with beta-blocker and angiotensin converting enzyme inhibitor. He was then transferred to the catheterization laboratory. Fluoroscopy documented normal opening and closing of both leaflets of the mechanical aortic valve (video 2). Coronary angiography was also performed that revealed a large well-defined intraluminal filling defect in the distal portion of a dominant Left Circumflex Artery (LCX), with an image consistent with a thrombus (Figure 2-A) (video 3). Left Posterior Descending Artery (PDA) looked to be occluded at its origin by the thrombus (Figure 2-B). The left Anterior Descending (LAD) artery (Figure 2-C), non-dominant Right Coronary Artery (RCA), and other parts of LCX were normal and free of atherosclerosis. We decided to continue the aforementioned medical management without any further coronary interventions. He was started on warfarin on the next day and INR level was stabilized at a value of 3 for the subsequent five days. After comprehensive discussion with the patient and full training, he was discharged on aspirin, warfarin, beta-blocker, and angiotensin-converting enzyme-inhibitor medications.

\section{Discussion}

Coronary embolism is a rare cause of MI, but should be considered in patients with predisposing factors, including mechanical prosthetic valves, infective endocarditis, atrial fibrillation, intra- cardiac shunts, cardiac tumors, and atrial and ventricular thrombus formation in the context of valvular diseases or dilated cardiomyopathy (1-3).

Despite technical improvements in prosthesis design and development of less thrombogenic materials, mechanical valve prostheses still carry a significant thromboembolic risk even with adequate anticoagulant therapy. Patients with caged ball or tilting disk valves have a higher risk compared to those with bileaflet valves. On the other hand, a mechanical valve in the aortic position has a lower risk compared to mitral valve prosthesis $(4,5)$. From this point of view, our patient had a favorable profile (bileaflet valve, aortic position), but the
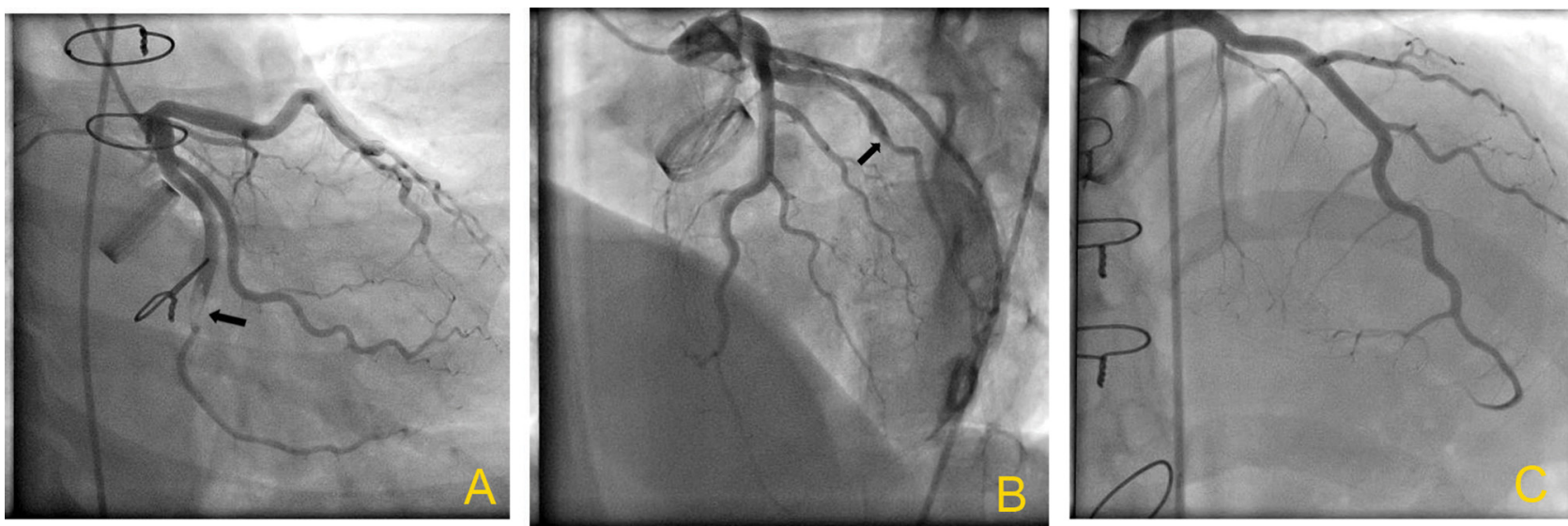

Figure 2. Coronary Angiogram, Which Revealed a Well-Defined Thrombus at Distal Portion of the Dominant Left Circumflex Coronary Artery (A), Left Posterior Descending Artery (PDA) Occluded at Its Origin by the Thrombus (B), Left Anterior Descending (C), and Right Coronary Arteries Free of Atherosclerosis 
major point is that he had recently discontinued anticoagulant therapy without any medical consultation.

The majority $(2,3)$ of emboli involves the LAD, which could be due to the preferential flow into the left coronary system related to aortic valve morphology or other hemodynamic factors that favor diastolic blood flow into the LAD. Coronary embolism into the LCX has been reported much less frequently compared to LAD, and RCA involvement is rare (6).

In cases of MI attributed to coronary thromboembolism, a source of thrombus formation is usually identified. In patients with a presumed embolic coronary event, TEE has been recommended to identify any potential source of emboli. However, it is known that even TEE has a low sensitivity to documenting the presence of micro-thrombi on a metallic valve. In some cases, as in the present case, no apparent thrombus is found (7). In such cases, a definite proof of an embolic etiology cannot be documented and, consequently, it remains a presumptive diagnosis. Our assumption regarding the embolic etiology in this patient was based on the predisposing factors for thrombosis (mechanical valve and interruption of anticoagulant therapy), clinical history suggestive of coincident cerebral micro-embolic event, and lack of atherosclerotic risk factors. Worth noting that our case was unique with respect to the concomitant cerebral transient ischemic attack. We did not find another similar case in the literature.

There is no consensus on the optimal management for coronary thromboembolism. Treatment options include intravenous or intracoronary thrombolytic therapy, percutaneous catheter aspiration embolectomy followed in some cases by balloon angioplasty with or without stenting, and medical management with anti-coagulants and IIb-IIIa inhibitors (8-11). Taniike et al. reported a case of nonSTEMI caused by bacterial embolism in proximal LCX that was treated with manual thrombectomy and stenting (12). Yuce et al. reported another case of non-STEMI as a result of thromboembolism in distal RCA in a patient with prosthetic mitral valve thrombosis.

They treated their patient medically with unfractionated heparin and tirofiban due to the distal location of thrombus (13). Likewise, we decided to manage our patient medically without coronary intervention considering the distal location of the thrombus with a relatively small territory, mild residual ST segment elevations, and inverted $\mathrm{T}$ waves on the ECG. Moreover, the patient's chest pain was subsided on admission.

What is crucial in patients with mechanical heart valves is the extreme importance of continued education and regular follow-up in order to prevent such life-threatening events. Overall, in individuals presenting with acute coronary syndrome, particularly those with mechanical valves, coronary emboli should be kept in mind, especially in unprotected, thrombotic state and absence of atherosclerotic risk factors

\section{Acknowledgements}

The authors would like to thank Dr. T. Moghiman for editing the manuscript.

\section{Authors' Contribution}

Each author has read and sufficiently contributed to the manuscript.

\section{Financial disclosure}

The authors declare that there is no conflict of interests.

\section{Funding/Support}

There is no funding/support.

\section{References}

1. Dagre A, Kouris N, Olympios C. Coronary artery disease: not always the case for ST-elevation myocardial infarction. J Invasive Cardiol. 2012;24(7):E142-4.

2. Levis JT, Schultz G, Lee PC. Acute Myocardial Infarction due to Coronary Artery Embolism in a Patient with a Tissue Aortic Valve Replacement. Perm J. 2011;15(3):82-6.

3. Nakazone MA, Tavares BG, Machado MN, Maia LN. Acute Myocardial Infarction due to Coronary Artery Embolism in a Patient with Mechanical Aortic Valve Prosthesis. Case Rep Med. 2010;2010:751857.

4. Cannegieter SC, Rosendaal FR, Wintzen AR, van der Meer FJ, Vandenbroucke JP, Briet E. Optimal oral anticoagulant therapy in patients with mechanical heart valves. NEngl J Med. 1995;333(1):11-7.

5. Kiernan TJ, Flynn AM, Kearney P. Coronary embolism causing myocardial infarction in a patient with mechanical aortic valve prosthesis. Int J Cardiol. 2006;112(2):e14-6.

6. Altinbas A, Kucuktepe Z, Ozaydin M, Aslan SM, Demir M, Altinbas E. A rare cause of myocardial infarction: acute inferoposterior myocardial infarction after successful intravenous thrombolytic treatment of mechanical mitral prosthetic valve thrombosis. Int $J$ Cardiovasc Imaging. 2005;21(5):509-12.

7. Yazici M, Kayrak M, Turan Y, Koc F, Ulgen MS. Acute coronary embolism without valve thrombosis in a patient with a prosthetic mitral valve--successful percutaneous coronary intervention: a case report. Heart Surg Forum. 2007;10(3):E228-30.

8. Karakoyun S, Gursoy MO, Kalcik M, Yesin M, Ozkan M. A case series of prosthetic heart valve thrombosis-derived coronary embolism. Turk Kardiyol Dern Ars. 2014;42(5):467-71.

9. Patel M, Bhangoo M, Prasad A. Successful percutaneous treatmen of suspected embolic left main thrombosis in a patient with a mechanical aortic valve. J Invasive Cardiol. 2011;23(11):E263-6.

10. Sial JA, Ferman MT, Saghir T, Rasool SI. Coronary embolism causing acute myocardial infarction in a patient with mitral valve prosthesis: successful management with angioplasty. J Pak Med Assoc. 2009;59(6):409-11.

11. Tang L, Hu XQ, Zhou SH. Coronary artery embolism causing acute myocardial infarction in patients with mechanical heart valve prosthesis: which is the optimal treatment? Heart Lung Circ. 2014;23(5):422-7.

12. Taniike M, Nishino M, Egami Y, Kondo I, Shutta R, Tanaka K, et al. Acute myocardial infarction caused by a septic coronary embolism diagnosed and treated with a thrombectomy catheter. Heart. 2005;91(5):e34.

13. Yuce M, Yavuz F, Cakici M, Sari I, Davutoglu V. A rare cause of myocardial infarction: coronary embolism in a patient with prosthetic mitral valve thrombosis. East Mediterr Health J. 2011;17(11):887-8. 